\title{
Valoración de la adherencia terapéutica de los pacientes de una unidad de hemodiálisis hospitalaria
}

\author{
Pilar Díaz de Argote Cervera, Sonia García Estévez, Patricia Arribas Cobo
}

Hospital Universitario Infanta Leonor. Madrid

\section{Introducción:}

Por adherencia terapéutica entendemos como el grado de conciencia del comportamiento de un paciente en relación con los medicamentos que ha de tomar, el seguimiento de una dieta o los cambios que ha de hacer en su estilo de vida, con las recomendaciones de los profesionales de salud que le atienden (1). La falta de adherencia terapéutica es un problema de salud pública con una prevalencia media del $50 \%$ en patologías crónicas y causalidad multifactorial (3). La Organización Mundial de la Salud (OMS) considera la falta de adherencia un tema prioritario de salud pública (4). El incumplimiento se puede ver favorecido por causas derivadas de la atención de los profesionales sanitarios, del propio paciente, de la enfermedad y derivadas del propio tratamiento (3) (5). Los métodos para medir la adherencia pueden ser directos o indirectos (2) (5). Siendo los indirectos más económicos y fáciles de aplicar (3) (6). La edad avanzada de nuestros pacientes, unida a la pluripatología asociada, conlleva a un consumo elevado de fármacos. El objetivo general de este trabajo es determinar el grado de cumplimiento terapéutico de los pacientes en nuestra unidad y estudiar que variables influyen en la no adherencia.

\section{Material y métodos:}

Estudio descriptivo de serie de casos en el que se pasó el cuestionario validado SMAQ sobre adherencia terapéutica. Incluimos 3 preguntas demográficas y 5 preguntas abiertas. Se considera paciente adherente, aquel que tiene un cumplimiento igual o superior al $95 \%$. Se estudiaron otras variables (sexo, edad, número de comprimidos y medicamentos prescritos, conocimiento de la utilidad del fármaco e influencia de costumbres). Las variables cualitativas se presentan con su distribución de frecuencias y las cuantitativas como media y desviación estándar. Las cuantitativas se correlacionan mediante chi-cuadrado o prueba exacta de Fisher. Se consideraron significativos $\mathrm{p}<0.05$.

\section{Resultados:}

Se estudiaron 64 pacientes; $60.9 \%$ hombres y $39.1 \%$ mujeres. Edad media $66,56 \pm 15$ años y tiempo mediano en diálisis 32.5 (RIQ 15-81) meses. El 62.5\% de los pacientes eran cumplidores con el tratamiento farmacológico. El $52 \%$ de las mujeres y el $28.2 \%$ de los hombres son cumplidores. El $42,5 \%$ de los pacientes conoce la medicación. El $21,7 \%$ se ve influido por las costumbres a la hora de tomar la medicación. Del 47,4 $\%$ de los pacientes que refería aceptar el tratamiento el $51,9 \%$ eran incumplidores. Del $52,6 \%$ de los que decían no aceptar su tratamiento el $80 \%$ eran incumplidores.

\section{Discusión:}

El porcentaje de cumplimiento de nuestros pacientes es mayor que la media descrita en la bibliografía para los pacientes crónicos, siendo las mujeres más cumplidoras que los hombres. Aquellos pacientes que dicen que no les influyen las costumbres son más incumplidores que aquellos que dicen que sí, pero no encontramos tampoco diferencias significativas y aquellos que afirmaban aceptar la medicación son significativamente más cumplidores. Los olvidos son la variable de mayor frecuencia en la definición de paciente no adherente $(41,3 \%)$. 
Aunque los cuestionarios son la herramienta más accesible como método indirecto para medir la adherencia terapéutica están supeditados a una gran subjetividad.

\section{Referencias Bibliográficas}

1. Palop Larrea V, Martínez Mir I. Adherencia al tratamiento del paciente anciano. Inf Ter Sis Nac Salud. 2004; 28 (5).

2. Servicio Madrileño de Salud. Gerencia de Atención Primaria, Áreas 1, 2, 3, 5 y 7. Notas farmacoterapéuticas. Adherencia terapéutica: estrategias prácticas de mejora. 2006; 13 (8).
3. Adherence to long-term therapies. Evidence for action. World Health Organization 2003.

4. Martínez MA, Callejo I. Cumplimiento terapéutico de los pacientes de hemodiálisis. Libro de Comunicaciones presentadas al XXXIV Congreso Nacional SEDEN. 2009. Pamplona.

5. http://www. fundapoyarte.org/contenidos/MET0DOS_INDIRECTOS_ADHERENCIA.pdf (Consultado el 23/03/13) 6. Sackett DL, Haynes RB, Gibson ES, Hackett BC, Taylor PW, Roberts RS. Randomised clinical trial of strategies for improving medication compliance in primary hypertension. The Lancet 1975;1:1205-7. 